\title{
Rifaximin Therapy for Patients with Irritable Bowel Syndrome without Constipation
}

\author{
Mark Pimentel, M.D., Anthony Lembo, M.D., William D. Chey, M.D., \\ Salam Zakko, M.D., Yehuda Ringel, M.D., Jing Yu, Ph.D., \\ Shadreck M. Mareya, Ph.D., Audrey L. Shaw, Ph.D., Enoch Bortey, Ph.D., \\ and William P. Forbes, Pharm.D., for the TARGET Study Group*
}

\section{A B STRACT}

From Cedars-Sinai Medical Center, Los Angeles (M.P.); Beth Israel Deaconess Medical Center and Harvard Medical School - both in Boston (A.L.); University of Michigan Health System, Ann Arbor (W.D.C.); Connecticut Gastroenterology Institute at Bristol Hospital, Bristol (S.Z.); University of North Carolina at Chapel Hill, Chapel Hill (Y.R.); and Salix Pharmaceuticals, Morrisville, NC (J.Y., S.M.M., A.L.S., E.B., W.P.F.). Address reprint requests to Dr. Forbes at $1700 \mathrm{Pe}$ rimeter Park Dr., Morrisville, NC 27560.

\footnotetext{
*Members of the TARGET Study Group are listed in the Supplementary Appendix, available at NEJM.org.
}

N Engl J Med 2011;364:22-32. Copyright @ 2011 Massachusetts Medical Society.

\section{BACKGROUND}

Evidence suggests that gut flora may play an important role in the pathophysiology of the irritable bowel syndrome (IBS). We evaluated rifaximin, a minimally absorbed antibiotic, as treatment for IBS.

\section{METHODS}

In two identically designed, phase 3, double-blind, placebo-controlled trials (TARGET 1 and TARGET 2), patients who had IBS without constipation were randomly assigned to either rifaximin at a dose of $550 \mathrm{mg}$ or placebo, three times daily for 2 weeks, and were followed for an additional 10 weeks. The primary end point, the proportion of patients who had adequate relief of global IBS symptoms, and the key secondary end point, the proportion of patients who had adequate relief of IBS-related bloating, were assessed weekly. Adequate relief was defined as selfreported relief of symptoms for at least 2 of the first 4 weeks after treatment. Other secondary end points included the percentage of patients who had a response to treatment as assessed by daily self-ratings of global IBS symptoms and individual symptoms of bloating, abdominal pain, and stool consistency during the 4 weeks after treatment and during the entire 3 months of the study.

\section{RESULTS}

Significantly more patients in the rifaximin group than in the placebo group had adequate relief of global IBS symptoms during the first 4 weeks after treatment ( $40.8 \%$ vs. $31.2 \%, \mathrm{P}=0.01$, in TARGET $1 ; 40.6 \%$ vs. $32.2 \%, \mathrm{P}=0.03$, in TARGET 2; $40.7 \%$ vs. $31.7 \%, \mathrm{P}<0.001$, in the two studies combined). Similarly, more patients in the rifaximin group than in the placebo group had adequate relief of bloating (39.5\% vs. $28.7 \%, \mathrm{P}=0.005$, in TARGET $1 ; 41.0 \%$ vs. $31.9 \%, \mathrm{P}=0.02$, in TARGET 2 ; $40.2 \%$ vs. $30.3 \%, \mathrm{P}<0.001$, in the two studies combined). In addition, significantly more patients in the rifaximin group had a response to treatment as assessed by daily ratings of IBS symptoms, bloating, abdominal pain, and stool consistency. The incidence of adverse events was similar in the two groups.

\section{CONCLUSIONS}

Among patients who had IBS without constipation, treatment with rifaximin for 2 weeks provided significant relief of IBS symptoms, bloating, abdominal pain, and loose or watery stools. (Funded by Salix Pharmaceuticals; ClinicalTrials.gov numbers, NCT00731679 and NCT00724126.) 
T THE IRRITABLE BOWEL SYNDROME (IBS) is a functional gastrointestinal disorder characterized by recurring symptoms of abdominal pain, bloating, and altered bowel function in the absence of structural, inflammatory, or biochemical abnormalities. ${ }^{1}$ IBS often does not respond to current treatment options, including dietary and lifestyle modifications, fiber supplementation, psychological therapy, and pharmacotherapy. ${ }^{2,3}$ Because no reliable biologic or structural markers have been identified, the effects of pharmacotherapy are typically assessed by asking patients to report whether they had adequate relief of IBS symptoms (with a binary response of yes or no). ${ }^{4}$ Given the limitations of available therapies, there is an unmet medical need for novel therapeutic approaches.

Patients with IBS may have alterations in the intestinal microbiota, ${ }^{5-7}$ thus leading investigators to consider targeting the intestinal microbiota for the treatment of this condition. Although some patients have had improvement with neomycin therapy, clinical trials have shown that it has marginal efficacy, and side effects limit the use of the drug. ${ }^{8}$ The use of systemic antibiotics has been reported with mixed results. ${ }^{9}$

Rifaximin (Xifaxan, Salix Pharmaceuticals) is an oral, nonsystemic, broad-spectrum antibiotic that targets the gut and is associated with a low risk of bacterial resistance. ${ }^{10-12}$ It has shown efficacy in small-scale studies of IBS. ${ }^{13,14}$ We present the results of two large-scale, identically designed, multicenter trials - TARGET 1 and TARGET $2-$ of 3 months' duration that examined the relief of IBS symptoms after a 2-week course of rifaximin.

\section{METHODS}

\section{STUDY PATIENTS}

Eligible patients were at least 18 years of age; had undergone a colonoscopic examination within the previous 2 years; had received a diagnosis of and had current symptoms of IBS (as assessed according to the Rome II diagnostic criteria for IBS ${ }^{15}$ ), in particular, symptoms of abdominal pain and discomfort; and did not have adequate relief of global IBS symptoms and of IBS-related bloating at both the time of screening and the time of randomization. Eligible patients rated the average daily amount of abdominal pain and of bloating as a score of 2 to 4.5 on a 7-point Likert scor- ing system (with 0 indicating not at all; 1, hardly; 2, somewhat; 3, moderately; 4, a good deal; 5, a great deal; and 6 , a very great deal) and rated the average daily consistency of their stools as 3.5 or more on a 5-point scale for stool consistency (with 1 indicating very hard; 2, hard; 3, formed; 4 , loose; and 5, watery) over the course of at least 7 days.

Exclusion criteria were constipation-predominant IBS (according to the definition in the Rome II criteria ${ }^{15}$ ), a history of inflammatory bowel disease, diabetes, unstable thyroid disease, previous abdominal surgery (other than cholecystectomy or appendectomy), human immunodeficiency virus infection, and renal or hepatic disease. Patients were excluded if they were currently taking alosetron, tegaserod, lubiprostone, warfarin, or antipsychotic, antispasmodic, antidiarrheal, probiotic, or narcotic drugs or if they had taken antibiotics within the previous 14 days or rifaximin within 60 days before signing the informed-consent form for the study. Patients were allowed to take antidepressant agents of the selective serotonin-reuptake inhibitor and tricyclic antidepressant classes, provided that they had been taking a stable dose for at least 6 weeks. All patients provided written informed consent before study-related procedures were initiated.

\section{STUDY DESIGN AND PROCEDURES}

The protocols were approved by the institutional review board or independent ethics committee at each center, and the studies were conducted in accordance with applicable laws and regulations (see the Supplementary Appendix, available with the full text of this article at NEJM.org). After a screening phase of 7 to 13 days, eligible patients were randomly assigned with the use of an interactive voice-response system to either rifaximin or placebo, in a 1:1 ratio. The randomization code was computer-generated, and randomization was performed in blocks of four, stratified according to center. After completing the 14-day studytreatment period, patients were evaluated for 10 additional weeks. Study visits were conducted on days $1,7,14,28$, and 84 , and patients were monitored by means of telephone calls on days 42,56 , and 70. Efficacy assessments were obtained daily by means of an interactive voice-response system over the course of the entire study (Fig. 1).

The protocol was designed by Salix Pharmaceuticals in collaboration with the academic au- 


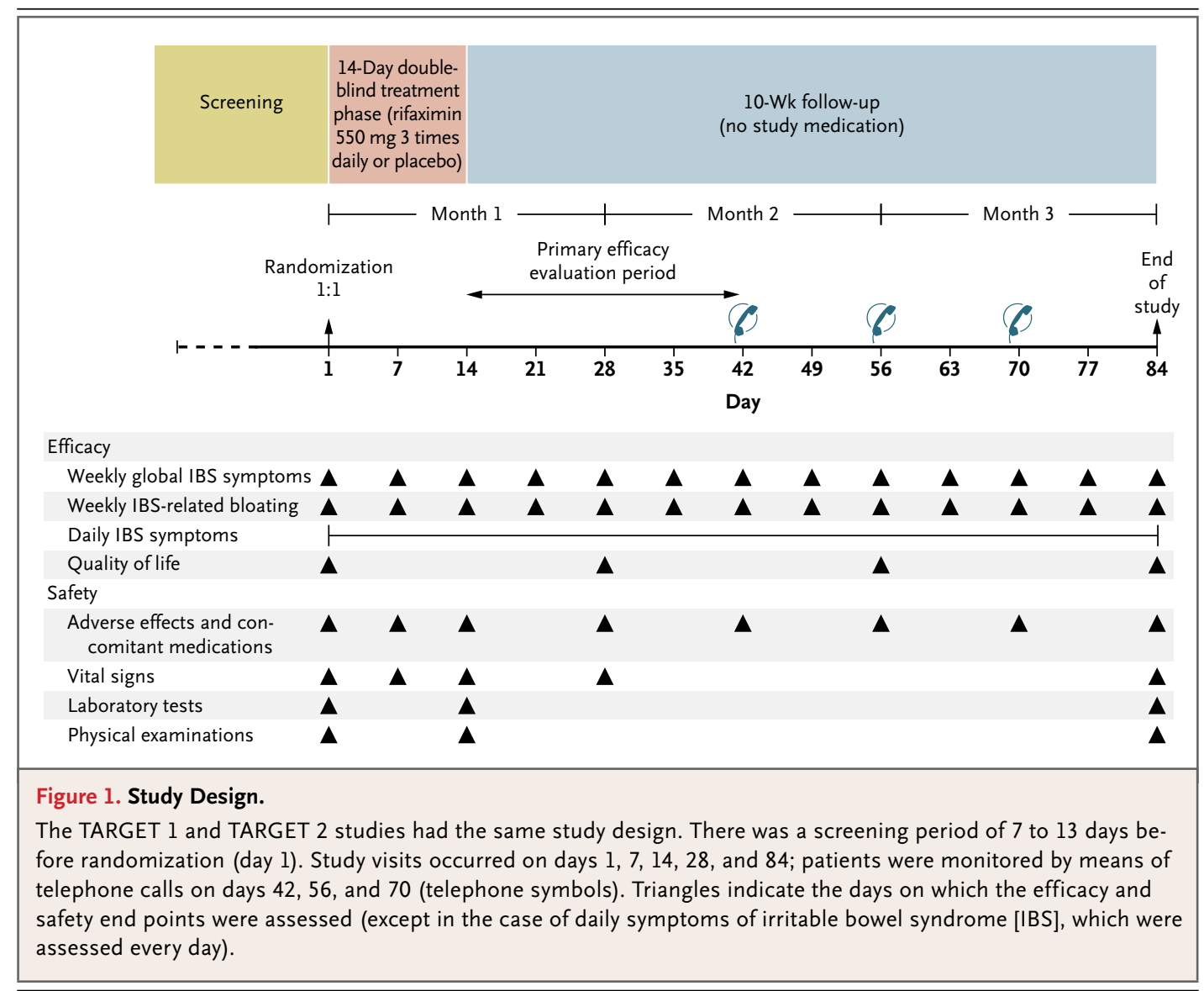

thors. Data were collected by investigators at each center (see the Supplementary Appendix) and were monitored by Quintiles (a contract research organization) under the supervision of Salix Pharmaceuticals. The data were analyzed by personnel at Salix Pharmaceuticals, in collaboration with the academic authors. All the authors participated in the interpretation of the data and in the writing of the manuscript. The first two authors wrote the first draft of the manuscript, and all the authors, in addition to an employee of Salix, assisted in the revision of subsequent drafts. All the authors made the decision to submit the manuscript for publication. The trial protocol, including the statistical analysis plan, is available at NEJM.org. All the authors vouch for the completeness and veracity of the data and analyses, as well as the fidelity of this report to the trial protocol.

\section{EFFICACY AND SAFETY END POINTS}

The primary end point was the proportion of patients who had adequate relief of global IBS symptoms for at least 2 of the 4 weeks during the primary evaluation period (weeks 3 through 6). This end point was determined from the response (yes or no) to the following question, which was asked weekly: "In regard to all your symptoms of IBS, as compared with the way you felt before you started the study medication, have you, in the past 7 days, had adequate relief of your IBS symptoms?" The threshold for clinical relevance adequate relief during at least 2 weeks per month - was defined prospectively. In addition, the proportion of patients who reported that they had adequate relief during at least 2 weeks per month ("monthly response") during months 1 , 2, and 3 was assessed to identify the onset and duration of the therapeutic effect. Patients who started to take antibiotics (other than the study medication) or who took more than two doses of a medication that was prohibited per the study protocol were considered not to have had a response to treatment starting from the time the medication was initiated, regardless of their response data. 
The key secondary end point, the proportion of patients who had adequate relief of IBS-related bloating during the primary evaluation period, was determined from the response (yes or no) to the weekly question, "In regard to your symptoms of bloating, as compared with the way you felt before you started study medication, have you, in the past 7 days, had adequate relief of your IBS symptom of bloating?" The onset and duration of relief of bloating were also assessed in an analysis of monthly response, as described above for the primary end point.

For other secondary end points, the proportion of patients who had relief was determined from the patients' daily assessments of IBS symptoms, bloating, and abdominal pain and discomfort (as rated on a 7-point scale); relief was defined as a score of either 0 (not at all) or 1 (hardly) for at least $50 \%$ of the days in a given week or a score of 0 (not at all), 1 (hardly), or 2 (somewhat) for $100 \%$ of the days in a given week for at least 2 of the 4 weeks during a given month.

An exploratory end point, which was included at the request of the Food and Drug Administration (FDA), was the proportion of patients who had relief of the composite of abdominal pain and discomfort and loose or watery stools (as measured by improvement in stool consistency), on the basis of daily assessments. Relief was defined as a decrease of at least 30\% from baseline in weekly mean ratings of IBS-related abdominal pain or discomfort and a weekly mean stoolconsistency score of less than 4 (with 4 indicating loose stools and lower scores indicating more formed stools) for at least 2 of the 4 weeks during a given month. This exploratory end point is consistent with the recommended composite end point for IBS with diarrhea in a recently released draft of FDA guidelines. ${ }^{16}$ Safety assessments included the monitoring of adverse events, results of clinical laboratory testing, findings from physical examinations, and vital signs.

\section{STATISTICAL ANALYSIS}

We estimated the sample size for each study assuming that $40 \%$ of the patients in the placebo group and $55 \%$ in the rifaximin group would meet the criteria for the primary end point (i.e., would have adequate relief of global IBS symptoms, as assessed weekly) for at least 2 of the first 4 weeks after treatment. With these assumptions, a sample of 300 patients would be needed in each group for the studies to have $95 \%$ power to show the 15-percentage-point difference between the groups, at a significance level of 0.05 .

All efficacy and safety analyses were performed on a modified intention-to-treat population, which included all patients who received at least one dose of the study drug. Missing data were imputed with the use of the last-observationcarried-forward method, whereby missing values were replaced with the last nonmissing value; baseline values were not carried forward. Two sensitivity analyses were conducted, one in which missing data were regarded as indicating that the patients who terminated the study prematurely had had no relief of symptoms, and the other in which missing data were imputed with the use of the multiple-imputation method.

Binary data (i.e., data on the proportion of patients who had or did not have adequate relief of symptoms) were analyzed with the use of logistic regression; fixed-effect terms included the study group and the analysis center. There were five analysis centers, which we formed prospectively by grouping the study centers according to geographic region in order to assess the effects of geographic location on the end points. For the analysis of ordinal data (i.e., data on the number of months in which patients had relief for at least 2 weeks per month), we used the proportional-odds model for the ordinal outcome. The number of consecutive months with relief during the first 3 months after treatment was summed for each patient, so that each patient received a score of $0,1,2$, or 3 .

We analyzed the changes from baseline in continuous outcomes (i.e., symptom scores) by fitting fixed-effects linear models to the data. An initial model with terms for treatment, analysis center, baseline ratings of the response variable, and interaction of baseline ratings with treatment was fitted. The interaction term was tested at the 0.05 level. A nonsignificant interaction was dropped from the model in subsequent analyses.

Spearman correlation analyses were applied to the mean change from baseline in daily assessments of adequate relief of IBS symptoms (yes or no) to determine whether the weekly assessments of adequate relief paralleled the pattern seen with the daily assessments. Safety data were summarized with the use of descriptive statistics. 


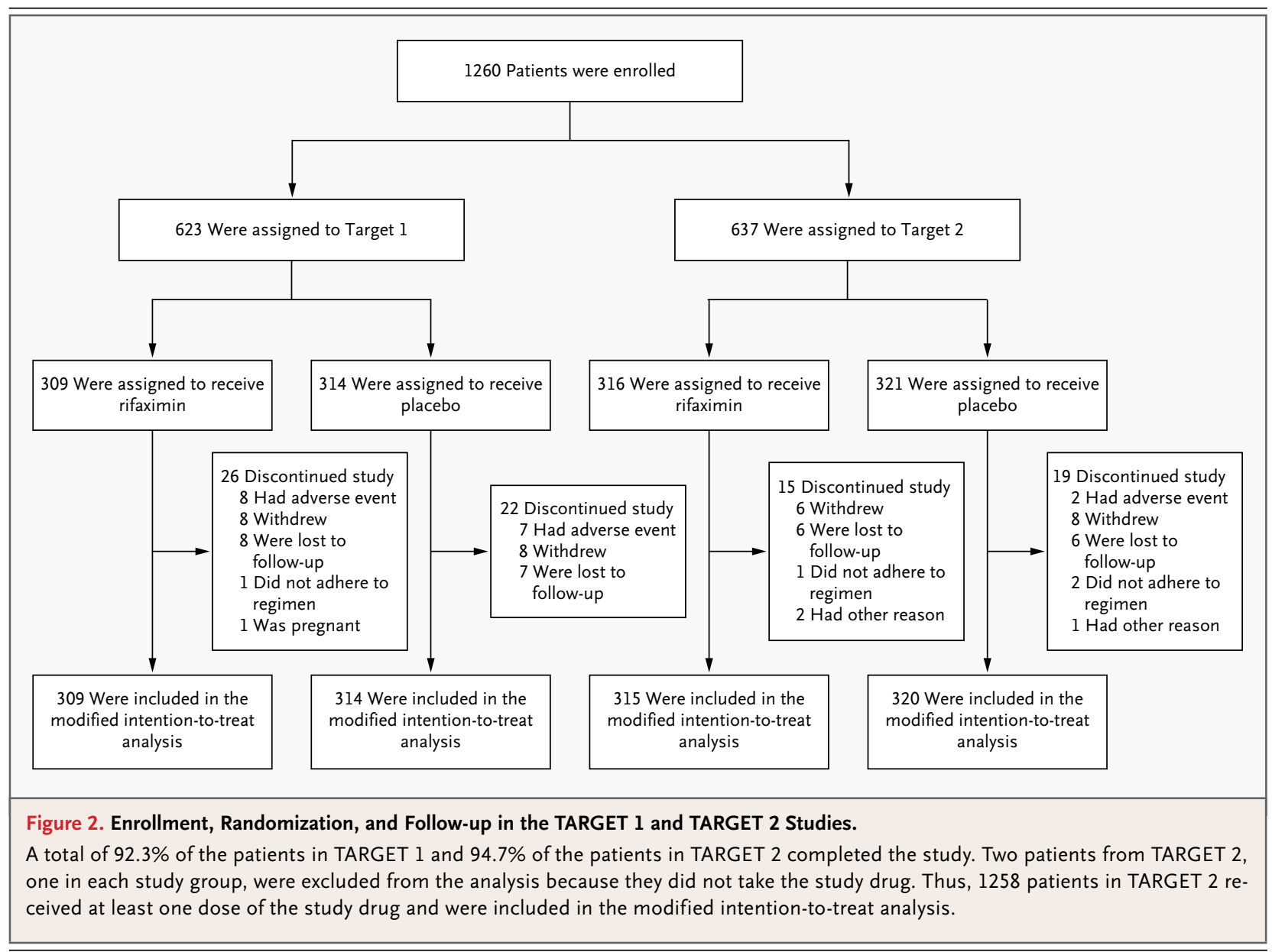

\section{RESULTS}

STUDY PATIENTS

A total of 1260 patients who had IBS without constipation were enrolled in the studies (623 patients in TARGET 1 and 637 in TARGET 2) and underwent randomization at one of 179 investigative sites in the United States (1217 patients) and Canada (43 patients) (Fig. 2). The studies were conducted in parallel from June 2008 through August 2009. In TARGET 1, all the patients who underwent randomization took at least one dose of the study drug. In TARGET 2, two patients (one in each group) underwent randomization but did not receive a study drug. Thus, 1258 patients received at least one dose of the study drug and were included in the modified intention-to-treat population. More than $90 \%$ of the patients completed the entire 12-week study. The baseline characteristics of the patients were similar in the two studies and across treatment groups (Table 1). The rate of adherence to the study drug, defined as the use of at least $70 \%$ of the dispensed tablets, was at least $97 \%$ in both study groups in both studies.

\section{EFFICACY DURING THE PRIMARY EVALUATION PERIOD (WEEKS 3 THROUGH 6)}

Adequate Relief of Global IBS Symptoms

Significantly more patients in the rifaximin group than in the placebo group met the criteria for the primary end point of adequate relief of global IBS symptoms for at least 2 of the first 4 weeks after treatment $(40.8 \%$ vs. $31.2 \%, \mathrm{P}=0.01$, in TARGET 1 ; $40.6 \%$ vs. $32.2 \%, \mathrm{P}=0.03$, in TARGET $2 ; 40.7 \%$ vs. $31.7 \%, \mathrm{P}<0.001$, in the two studies combined) (Fig. 3A). The last-observation-carried-forward method was applied in the case of $5.8 \%$ of the patients in the rifaximin group and $5.7 \%$ of the patients in the placebo group in TARGET 1 and in the case of $5.4 \%$ in the rifaximin group and $8.4 \%$ in the placebo group in TARGET 2. Overall, 


\begin{tabular}{|c|c|c|c|c|c|c|}
\hline \multirow[t]{2}{*}{ Characteristic } & \multicolumn{3}{|c|}{ TARGET 1} & \multicolumn{3}{|c|}{ TARGET 2} \\
\hline & $\begin{array}{l}\text { Rifaximin } \\
(N=309)\end{array}$ & $\begin{array}{l}\text { Placebo } \\
(\mathrm{N}=314)\end{array}$ & P Value' & $\begin{array}{l}\text { Rifaximin } \\
(\mathrm{N}=315)\end{array}$ & $\begin{array}{l}\text { Placebo } \\
(\mathrm{N}=320)\end{array}$ & P Value'† \\
\hline Age $-y r$ & $46.2 \pm 15.0$ & $45.5 \pm 14.6$ & 0.55 & $45.9 \pm 13.9$ & $46.3 \pm 14.6$ & 0.72 \\
\hline Age group — no. (\%) & & & 0.71 & & & 0.44 \\
\hline$<65 \mathrm{yr}$ & $275(89.0)$ & $276(87.9)$ & & $285(90.5)$ & $283(88.4)$ & \\
\hline$\geq 65 \mathrm{yr}$ & $34(11.0)$ & $38(12.1)$ & & $30(9.5)$ & $37(11.6)$ & \\
\hline Sex - no. (\%) & & & 0.15 & & & 0.66 \\
\hline Male & $74(23.9)$ & $92(29.3)$ & & $88(27.9)$ & $95(29.7)$ & \\
\hline Female & $235(76.1)$ & $222(70.7)$ & & $227(72.1)$ & $225(70.3)$ & \\
\hline Race - no. $(\%) \div$ & & & 0.50 & & & 0.08 \\
\hline Black or of African ancestry & $24(7.8)$ & $30(9.6)$ & & $21(6.7)$ & $14(4.4)$ & \\
\hline White & $281(90.9)$ & $280(89.2)$ & & $282(89.5)$ & $302(94.4)$ & \\
\hline Other & $4(1.3)$ & $4(1.3)$ & & $12(3.8)$ & $4(1.2)$ & \\
\hline \multicolumn{7}{|l|}{ Average daily scores } \\
\hline 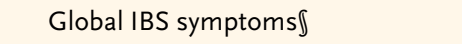 & $3.4 \pm 0.7$ & $3.4 \pm 0.7$ & 0.47 & $3.4 \pm 0.7$ & $3.4 \pm 0.7$ & 0.53 \\
\hline IBS-related bloating $\mathbb{}$ & $3.3 \pm 0.8$ & $3.3 \pm 0.7$ & 0.43 & $3.2 \pm 0.7$ & $3.3 \pm 0.7$ & 0.28 \\
\hline Abdominal pain and discomfort $\rrbracket$ & $3.3 \pm 0.7$ & $3.2 \pm 0.7$ & 0.75 & $3.3 \pm 0.7$ & $3.3 \pm 0.7$ & 0.69 \\
\hline Stool consistency 9 & $3.9 \pm 0.3$ & $3.9 \pm 0.3$ & 0.18 & $3.9 \pm 0.3$ & $3.9 \pm 0.3$ & 0.80 \\
\hline Average daily bowel movements - no. & $2.9 \pm 1.3$ & $3.0 \pm 1.4$ & 0.27 & $3.0 \pm 1.6$ & $3.0 \pm 1.5$ & 0.78 \\
\hline Days with stool urgency — \%\| & $81.8 \pm 22.3$ & $82.9 \pm 22.3$ & 0.53 & $81.3 \pm 22.8$ & $82.2 \pm 22.5$ & 0.64 \\
\hline Duration of IBS symptoms - yr*** & $11.9 \pm 10.5$ & $11.4 \pm 11.9$ & 0.61 & $10.8 \pm 10.2$ & $11.8 \pm 10.4$ & 0.19 \\
\hline \multicolumn{7}{|c|}{$\begin{array}{l}\text { Plus-minus values are means } \pm \text { SD. IBS denotes irritable bowel syndrome. } \\
\mathrm{P} \text { values were calculated with the use of a t-test for continuous variables and Fisher's exact test for nominal variables. } \\
\text { Race was self-reported. } \\
\text { Patients were asked to rate how bothersome their symptoms were on that day, on a scale of } 0 \text { to } 6 \text {. A score of } 0 \text { indicated not at all; } 1 \text {, } \\
\text { hardly; } 2 \text {, somewhat; } 3 \text {, moderately; } 4 \text {, a good deal; } 5 \text {, a great deal; and } 6 \text {, a very great deal. } \\
\text { Patients were asked to rate the overall stool form of their bowel movements on that day. A score of } 1 \text { indicated very hard; } 2 \text {, hard; } 3 \text {, } \\
\text { formed; } 4 \text {, loose; and } 5 \text {, watery. } \\
\text { The percentage of days with stool urgency was calculated with the use of the following formula: } 100 \times \text { (no. of days with a sense of urgency } \\
\text { with any of the bowel movements } \div \text { no. of days with bowel movements). } \\
\text { The duration of IBS symptoms was calculated with the use of the following formula: (date written informed consent was provided - date of } \\
\text { first IBS symptom) } \div 365.25 \text {. }\end{array}$} \\
\hline
\end{tabular}

sensitivity analyses that were performed to address the effect of missing values yielded results that were consistent with those of the primary efficacy analysis (see the Supplementary Appendix). On the basis of daily assessments of IBS symptoms, the proportion of patients with a response to treatment, as rated on a 7-point scale during the primary evaluation period, was significantly greater in the rifaximin group than in the placebo group $(42.7 \%$ vs. $30.6 \%, \mathrm{P}<0.001$, in TARGET $1 ; 37.8 \%$ vs. $28.4 \%, \mathrm{P}=0.007$, in TARGET $2 ; 40.2 \%$ vs. $29.5 \%, \mathrm{P}<0.001$, in the two studies combined) (Fig. 3A).

\section{Adequate Relief of IBS-Related Bloating}

Significantly more patients in the rifaximin group than in the placebo group met the criteria for the key secondary end point, adequate relief of IBSrelated bloating for at least 2 of the first 4 weeks after treatment $(39.5 \%$ vs. $28.7 \%, P=0.005$, in TARGET $1 ; 41.0 \%$ vs. $31.9 \%, \mathrm{P}=0.02$, in TARGET $2 ; 40.2 \%$ vs. $30.3 \%, P<0.001$, in the two studies combined) (Fig. 3A). Sensitivity analyses that were performed to assess the effect of missing values yielded similar results (see the Supplementary Appendix). On the basis of daily assessments of IBSrelated bloating as rated on a 7-point scale during 


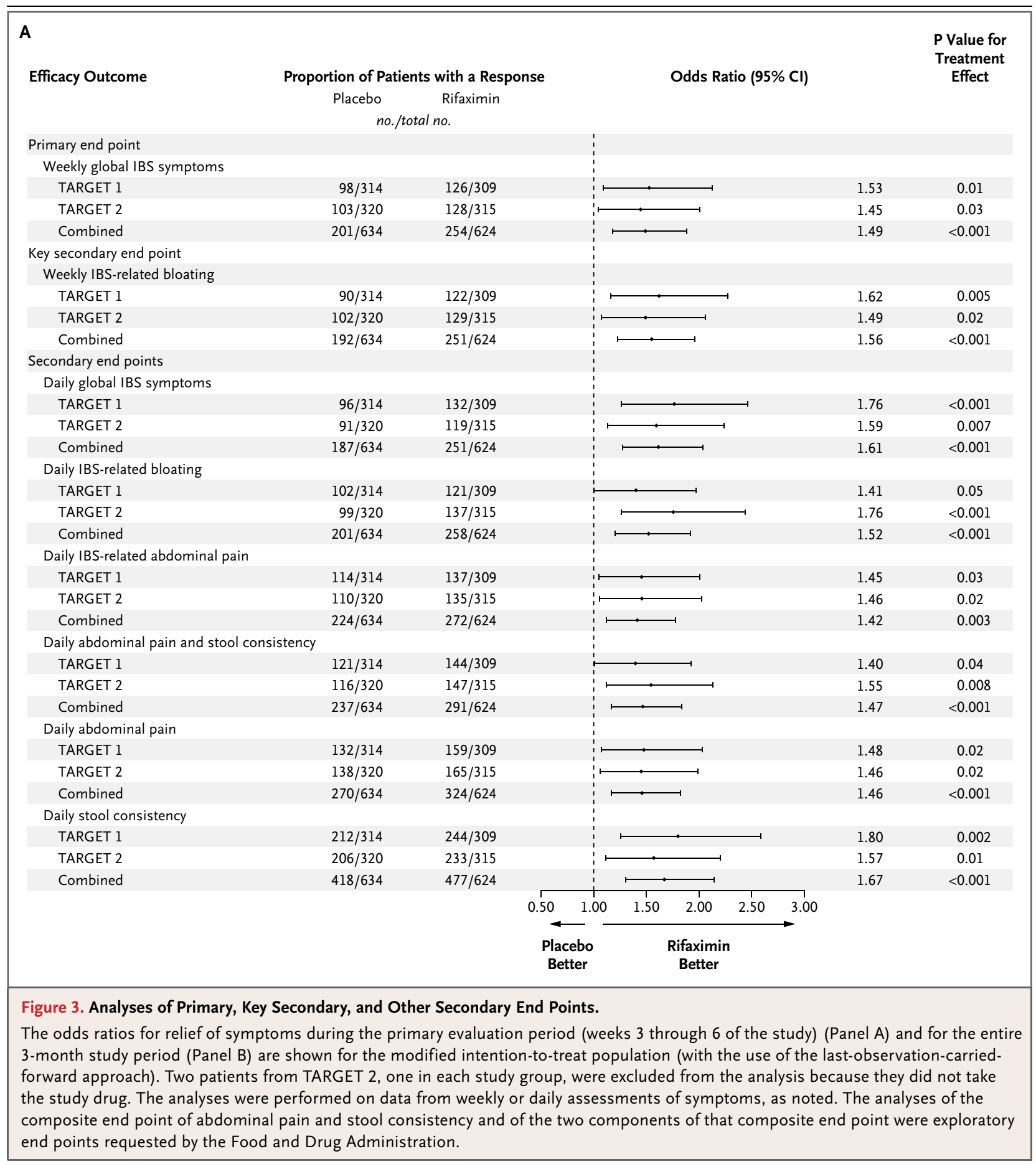

the same primary evaluation period, a significant- Relief of IBS-Related Abdominal Pain and Loose ly greater proportion of patients in the rifaximin or Watery Stools

group than in the placebo group had relief $339.2 \%$ A significantly greater proportion of patients in vs. $32.5 \%, \mathrm{P}=0.05$, in TARGET $1 ; 43.5 \%$ vs. $30.9 \%$, the rifaximin group than in the placebo group $\mathrm{P}<0.001$, in TARGET $2 ; 41.3 \%$ vs. $31.7 \%, \mathrm{P}<0.001$, had relief of IBS-related abdominal pain and disin the two studies combined) (Fig. 3A).

comfort during the primary evaluation period 


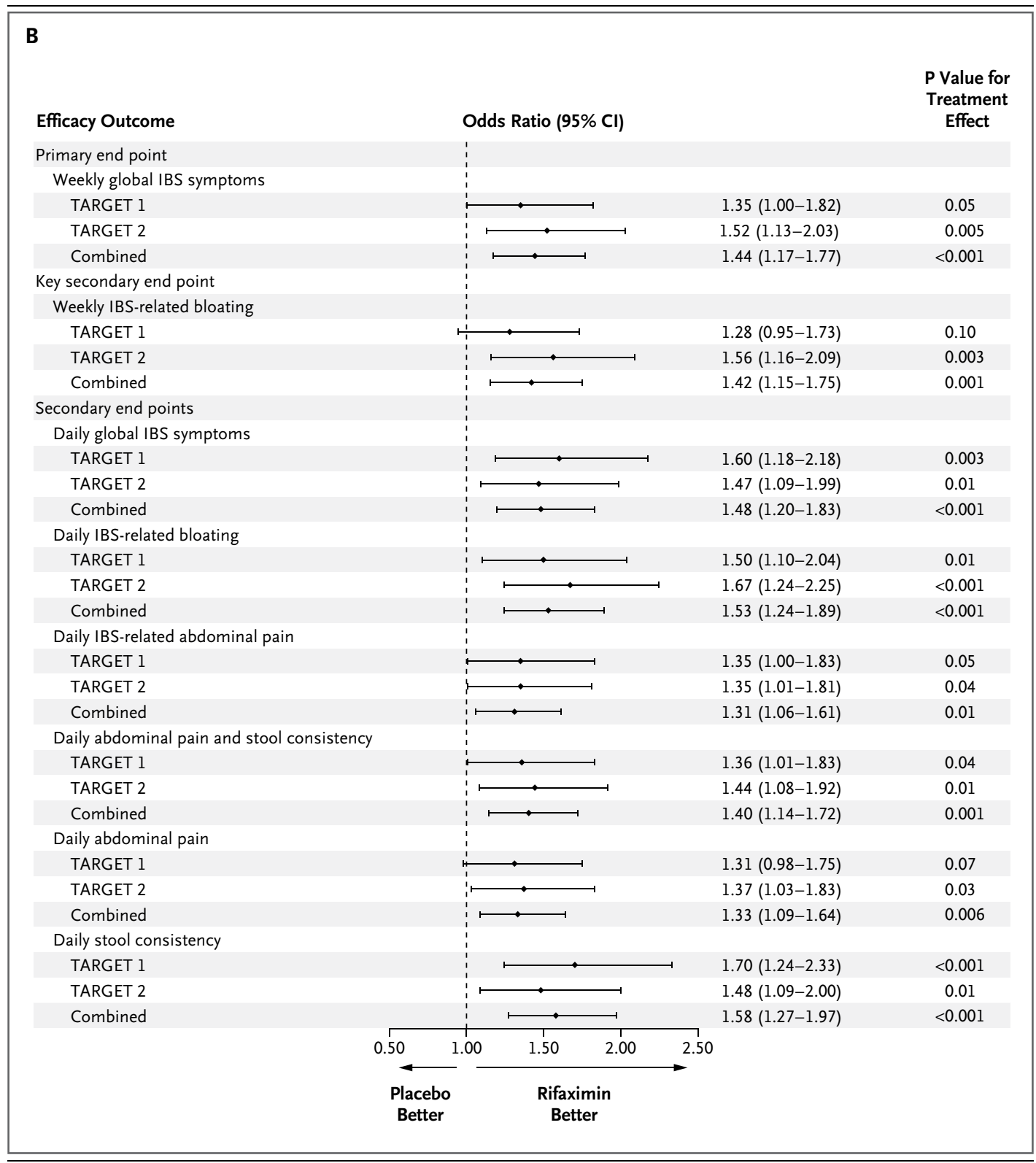

(44.3\% vs. $36.3 \%, \mathrm{P}=0.03$, in TARGET $1 ; 42.9 \%$ vs. $34.4 \%, \mathrm{P}=0.02$, in TARGET 2) (Fig. $3 \mathrm{~A}$ ). In an assessment of the composite end point of abdominal pain or discomfort and loose or watery stools, significantly more patients in the rifaximin group than in the placebo group had relief during the primary evaluation period (46.6\% vs. $38.5 \%, \mathrm{P}=0.04$, in TARGET $1 ; 46.7 \%$ vs. $36.3 \%$, $\mathrm{P}=0.008$, in TARGET 2 ), and a significantly greater proportion of patients in the rifaximin group had relief with respect to the individual components of this end point (Fig. 3A).

\section{EFFICACY DURING THE ENTIRE STUDY PERIOD} (MONTHS I THROUGH 3)

Durability of Response on the Basis of Weekly Assessment

In analyses of the monthly response evaluated on the basis of weekly assessments, more patients in the rifaximin group than in the placebo group in both studies had adequate relief of global IBS symptoms within the first month, with continued relief during the first 2 months and during all 3 months in both studies $(P=0.05$ in TARGET 1 , $\mathrm{P}=0.005$ in TARGET 2 , and $\mathrm{P}<0.001$ in the two 


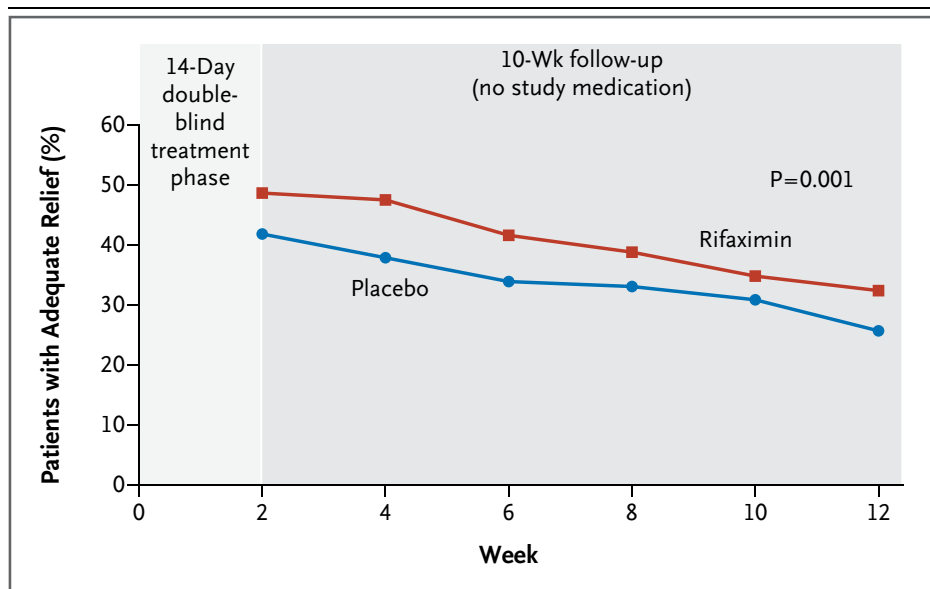

Figure 4. Percentage of Patients with Adequate Relief of Global IBS Symptoms in the TARGET 1 and TARGET 2 Studies Combined.

Adequate relief was defined as self-reported relief from symptoms for at least 1 week of every 2-week period. The $P$ value was calculated on the basis of a longitudinal data analysis with the use of a generalized-estimatingequation model, with fixed effects of treatment, analysis center, and week. Similar figures for the individual TARGET 1 and TARGET 2 trials are shown in the Supplementary Appendix. studies combined, for relief during all 3 months) (Fig. 3B). With respect to IBS-related bloating, in TARGET 1 , significantly more patients in the rifaximin group than in the placebo group had adequate relief in the first month, with continued relief during the first 2 months, but there were no significant between-group differences with respect to relief during all 3 months; in TARGET 2, significant benefits of rifaximin were seen with respect to IBS-related bloating for all 3 months $(\mathrm{P}=0.10$ in TARGET $1, \mathrm{P}=0.003$ in TARGET 2 , and $\mathrm{P}=0.001$ in the two studies combined, for relief during all 3 months) (Fig. 3B). The percentages of patients in each group with adequate relief of global IBS symptoms in TARGET 1 and TARGET 2 are provided in Figure 4, and in Figure $1 \mathrm{~A}$ and $1 \mathrm{~B}$ in the Supplementary Appendix.

Durability of Response on the Basis of Daily Assessment The analyses of monthly response evaluated on the basis of daily assessments also support a durable response to rifaximin in patients with IBS over the course of 3 months. Patients treated with rifaximin, as compared with patients who received placebo, had adequate relief of global IBS symptoms during the entire 3 months of the study $(\mathrm{P}=0.003$ in TARGET $1, \mathrm{P}=0.01$ in TARGET 2 , and $\mathrm{P}<0.001$ in the two studies combined) and of IBS-related bloating $(\mathrm{P}=0.01$ in TARGET 1 ,
$\mathrm{P}<0.001$ in TARGET 2 , and $\mathrm{P}<0.001$ in the two studies combined) (Fig. 3B).

An analysis of the monthly response evaluated on the basis of daily assessments of IBS-related abdominal pain and discomfort showed that significantly more patients in the rifaximin group than in the placebo group had relief for the entire 3 months $(\mathrm{P}=0.05$ in TARGET $1, \mathrm{P}=0.04$ in TARGET 2 , and $\mathrm{P}=0.01$ in the two studies combined) (Fig. 3B). An analysis of the monthly response evaluated on the basis of the assessment of the composite end point of abdominal pain and stool consistency also showed a significant benefit with rifaximin as compared with placebo $(\mathrm{P}=0.04$ in TARGET $1, \mathrm{P}=0.01$ in TARGET 2 , and $\mathrm{P}=0.001$ in the two studies combined) (Fig. 3B).

The mean improvement from baseline in daily symptom scores (global IBS symptoms, IBS-related bloating, IBS-related abdominal pain or discomfort, stool consistency, and the percentage of days with stool urgency) was greater for the patients who received rifaximin than for the patients who received placebo (Fig. 2 through 6 in the Supplementary Appendix).

\section{RESPONSIVENESS AND CONSTRUCT VALIDITY OF PATIENT-REPORTED OUTCOMES}

The response of patients with respect to adequate relief of global IBS symptoms and of IBS-related bloating was consistent with the response with respect to other IBS-related assessments. In addition, patients who had adequate relief of global IBS symptoms and of IBS-related bloating had greater improvements in daily symptom-severity scores than did patients who did not have adequate relief, regardless of study group, during each week in each study $(\mathrm{P}<0.001)$.

We tested the validity of using assessments of global IBS symptoms to measure changes in IBS symptoms by examining the correlation between these measures and changes in daily severity scores and bowel function; the results support the validity and usefulness of the primary end point of global IBS symptoms. Evidence for convergent validity (Spearman's correlation of 0.40 or higher) was observed between weekly adequate relief of global IBS symptoms and measures of daily symptom severity and bowel function.

\section{SAFETY}

The safety profile of rifaximin was similar to that of placebo (Table 2). Serious adverse events 
were recorded in 10 patients in the rifaximin group (1.6\%) and 15 patients in the placebo group (2.4\%). There were no cases of Clostridium difficileassociated diarrhea or ischemic colitis. No deaths were reported.

\section{I S C USSION}

Treating IBS is important because the symptoms cause substantial impairment in health-related quality of life, leading to increased use of health resources and reduced work productivity. ${ }^{17-20}$ These two phase 3 studies showed that a short course of rifaximin leads to sustained amelioration of the symptoms of IBS without constipation in a subgroup of affected patients.

The antibiotic effect of rifaximin is the presumed mechanism for its sustained beneficial effects in patients with IBS. A response to antibiotic therapy in patients with IBS has been shown to correlate with normalization of the results of lactulose hydrogen breath tests. ${ }^{8,13}$ However, there is debate about which antibioticrelated effect is most important. On the basis of existing data, there are three reasonable explanations: rifaximin affects gut bacteria and reduces bacterial products that negatively affect the host, the effect on gut flora reduces local mucosal engagement of bacteria such as the immune responses of the host, or the antibiotic alters both the bacteria and the host response. Whatever the final pathway, the durable effects suggest that rifaximin is affecting an underlying cause of IBS that is linked to an alteration in the intestinal microbiota. ${ }^{6,7,21,22}$ Some patients in both of our studies did not have a response to treatment, a finding that is consistent with the results of other placebo-controlled clinical trials involving patients with IBS ${ }^{23-25}$ and that may reflect differences in the underlying cause of the symptoms.

Similar percentages of patients in the rifaximin group and in the placebo group had adverse events. In this short-term study, the incidence of infections was similar in the two groups, and there were no cases of $C$. difficile-associated diarrhea or ischemic colitis.

In summary, the results of these two phase 3 studies showed that treatment with rifaximin at a dose of $550 \mathrm{mg}$ three times daily for 14 days provides better relief of symptoms of IBS than does placebo for up to 10 weeks after completion of therapy.
Table 2. Adverse Events during the 12-Week Study.*

$\begin{array}{ccc}\text { Event } & \begin{array}{c}\text { Rifaximin } \\ (\mathrm{N}=624)\end{array} & \begin{array}{c}\text { Placebo } \\ (\mathrm{N}=634)\end{array} \\ & \text { number (percent) }\end{array}$

\section{Adverse events}

$\begin{array}{lcr}\text { Headache } & 38(6.1) & 42(6.6) \\ \text { Upper respiratory tract infection } & 35(5.6) & 39(6.2) \\ \text { Abdominal pain } & 29(4.6) & 35(5.5) \\ \text { Nausea } & 27(4.3) & 24(3.8) \\ \text { Diarrhea } & 27(4.3) & 22(3.5) \\ \text { Nasopharyngitis } & 19(3.0) & 34(5.4) \\ \text { Sinusitis } & 17(2.7) & 16(2.5) \\ \text { Vomiting } & 15(2.4) & 9(1.4) \\ \text { Bronchitis } & 13(2.1) & 17(2.7) \\ \text { Cough } & 13(2.1) & 9(1.4) \\ \text { Flatulence } & 10(1.6) & 14(2.2) \\ \text { Back pain } & 10(1.6) & 15(2.4) \\ \text { Pharyngolaryngeal pain } & 9(1.4) & 15(2.4) \\ \text { Serious adverse events: } & & \\ \text { Chest pain } & 1(0.2) & 2(0.3) \\ \text { Breast cancer } & 1(0.2) & 1(0.2) \\ \text { Cholecystitis or acute cholecystitis } & 0 & 2(0.3) \\ \text { Adverse } & & \end{array}$

Adverse events possibly related to infection $\int$

$\begin{array}{lrr}\text { Upper respiratory tract infection } & 35(5.6) & 39(6.2) \\ \text { Viral gastroenteritis } & 7(1.1) & 8(1.3) \\ \text { Gastroenteritis } & 6(1.0) & 3(0.5) \\ \text { Cellulitis } & 3(0.5) & 1(0.2) \\ \text { Pneumonia } & 1(0.2) & 5(0.8) \\ \text { Viral infection } & 1(0.2) & 4(0.6)\end{array}$

* Values are pooled data from the TARGET 1 and TARGET 2 studies. Adverse events are listed in descending order of frequency in the rifaximin group.

$\uparrow$ The adverse events listed were reported in $2 \%$ or more of the patients in either treatment group.

$\checkmark$ The serious adverse events listed were reported in at least 2 of the patients in any treatment group.

$\int$ The listed adverse events possibly related to infections are known side effects of the use of systemic antibiotics as a drug class, as well as known effects of rifaximin.

Supported by Salix Pharmaceuticals.

Dr. Pimentel reports receiving grant support (to his institution) and consulting fees and honoraria from Salix Pharmaceuticals and reports that Cedars-Sinai Medical Center holds patents licensed by Salix Pharmaceuticals; Dr. Lembo reports receiving consulting fees from Salix Pharmaceuticals, Ironwood Pharmaceuticals, Prometheus, Ardelyx, Theravance, GlaxoSmithKline, and AstraZeneca, receiving payment for participation in several advisory-board meetings relating to the development of rifaximin for the treatment of IBS, and receiving payment as a member of the speakers' bureau of Salix Pharmaceuticals; Dr. Chey reports receiving consulting fees and honoraria from 
Procter \& Gamble, Ortho-McNeil, Prometheus, and Salix Pharmaceuticals, payment for the development of educational presentations, and payment or reimbursement for travel and accommodations from Procter \& Gamble, Prometheus, and Salix Pharmaceuticals; Dr. Zakko reports receiving grant support (to his institution), consulting fees, payment for the development of educational presentations, and payment or reimbursement for travel and accommodations from Salix Pharmaceuticals and holding stock in Salix Pharmaceuticals; Dr. Ringel reports receiving consulting fees from Salix Pharmaceuticals, Ironwood Pharmaceuticals, Procter \& Gamble, Pfizer, and Danisco, participating in several advisory-board meetings related to the development of rifaximin for the treatment of IBS, receiving payment for the development of educational presentations and as a member of the speakers' bureau of Salix Pharmaceuticals, and receiving research grants from Danisco USA, General Mills, and SmartPill. Drs. Yu, Mareya, and Bortey report being employees of and holding stock in Salix Pharmaceuticals; Dr. Shaw reports being an employee of Salix Pharmaceuticals and reports that she holds stock in the company; and Dr. Forbes reports being an officer and employee of Salix Pharmaceuticals and holding stock in the company. No other potential conflict of interest relevant to this article was reported.

Disclosure forms provided by the authors are available with the full text of this article at NEJM.org.

We thank Kristofer Klein, M.S. (Salix Pharmaceuticals), for assistance with the preparation of the manuscript.

\section{REFERENCES}

1. Brandt LJ, Chey WD, Foxx-Orenstein $\mathrm{AE}$, et al. An evidence-based systematic review on the management of irritable bowel syndrome. Am J Gastroenterol 2009; 104:Suppl 1:S1-S35.

2. Cash $\mathrm{BD}$, Chey WD. Advances in the management of irritable bowel syndrome. Curr Gastroenterol Rep 2003;5:468-75.

3. Schoenfeld P. Efficacy of current drug therapies in irritable bowel syndrome: what works and does not work. Gastroenterol Clin North Am 2005;34:319-35.

4. Spiegel B, Camilleri M, Bolus R, et al. Psychometric evaluation of patient-reported outcomes in irritable bowel syndrome randomized controlled trials: a Rome Foundation report. Gastroenterology 2009; 137:1944-53.

5. Ringel Y, Carroll IM. Alterations in the intestinal microbiota and functional bowel symptoms. Gastrointest Endosc Clin N Am 2009;19:141-50.

6. Posserud I, Stotzer P, Björnsson E, Abrahamsson H, Simrén M. Small intestinal bacterial overgrowth in patients with irritable bowel syndrome. Gut 2007;56: 802-8.

7. Kassinen A, Krogius-Kurikka L, Makivuokko $\mathrm{H}$, et al. The fecal microbiota of irritable bowel syndrome patients differs significantly from that of healthy subjects. Gastroenterology 2007;133:24-33.

8. Pimentel M, Chow EJ, Lin HC. Normalization of lactulose breath testing correlates with symptom improvement in irritable bowel syndrome: a double-blind, randomized, placebo-controlled study. Am J Gastroenterol 2003;98:412-9.

9. Attar A, Flourié B, Rambaud J-C,
Franchisseur C, Ruszniewski P, Bouhnik Y. Antibiotic efficacy in small intestina bacterial overgrowth-related chronic diarrhea: a crossover, randomized trial. Gastroenterology 1999;117:794-7.

10. Gerard L, Garey KW, DuPont HL. Rifaximin, a nonabsorbable rifamycin antibiotic for use in nonsystemic gastrointestinal infections. Expert Rev Anti Infect Ther 2005;3:201-11.

11. Jiang ZD, DuPont HL. Rifaximin: in vitro and in vivo antibacterial activity a review. Chemotherapy 2005;51:Suppl: $67-72$

12. Debbia EA, Maioli E, Roveta S, Marchese A. Effects of rifaximin on bacteria virulence mechanisms at supra- and subinhibitory concentrations. J Chemother 2008;20:186-94.

13. Sharara AI, Aoun E, Abdul-Baki H Mounzer R, Sidani S, Elhajj I. A randomized double-blind placebo-controlled trial of rifaximin in patients with abdomina bloating and flatulence. Am J Gastroenterol 2006;101:326-33.

14. Pimentel M, Park S, Mirocha J, Kane SV, Kong Y. The effect of a nonabsorbed oral antibiotic (rifaximin) on the symptoms of the irritable bowel syndrome a randomized trial. Ann Intern Med 2006 145:557-63.

15. Thompson WG, Longstreth GF, Drossman DA, Heaton KW, Irvine EJ, MüllerLissner SA. Functional bowel disorders and functional abdominal pain. Gut 1999 45:Suppl 2:1143-7.

16. Guidance for industry: irritable bowe syndrome: clinical evaluation of products for treatment. Silver Spring, MD: Food and Drug Administration Center for Drug Evaluation and Research (CDER) and Center for Biologics Evaluation and Research (CBER), March 2010.

17. Drossman DA, Li Z, Andruzzi E, et al. U.S. householder survey of functional gastrointestinal disorders: prevalence, sociodemography, and health impact. Dig Dis Sci 1993;38:1569-80.

18. Camilleri M, Andresen V. Current and novel therapeutic options for irritable bowel syndrome management. Dig Liver Dis 2009;41:854-62.

19. Hammerle CW, Surawicz CM. Updates on treatment of irritable bowel syndrome. World J Gastroenterol 2008;14: 2639-49.

20. El-Serag HB. Impact of irritable bowel syndrome: prevalence and effect on healthrelated quality of life. Rev Gastroenterol Disord 2003;3:Suppl 2:S3-S11.

21. Pimentel M. Review of rifaximin as treatment for SIBO and IBS. Expert Opin Investig Drugs 2009;18:349-58.

22. Brigidi P, Swennen E, Rizzello F, Bozzolasco M, Matteuzzi D. Effects of rifaximin administration on the intestinal microbiota in patients with ulcerative colitis. J Chemother 2002;14:290-5.

23. Lotronex (alosetron hydrochloride) tablets. San Diego, CA: Prometheus Laboratories, 2008 (package insert).

24. Zelnorm (tegaserod maleate) tablets. East Hanover, NJ: Novartis Pharmaceuticals, 2007 (package insert).

25. Amitiza (lubiprostone) capsules. Deerfield, IL: Takeda Pharmaceuticals America, 2008 (package insert).

Copyright (c) 2011 Massachusetts Medical Society. 